\title{
Flujos migratorios de China en el estado de Puebla, México
}

DOI: $10.32870 /$ mycp.v3i8.464

Juan Carlos Gachúz Maya ${ }^{1}$

\section{Resumen}

El objetivo general de este artículo de investigación es analizar de manera cualitativa las principales razones y causas de los flujos migratorios de China al estado de Puebla en los últimos 15 años. China se ha convertido en uno de los principales socios comerciales de nuestro país y la población china en México representa una de las comunidades de migrantes con mayor crecimiento; de manera reciente Puebla se ha convertido en uno de los principales destinos de migraciones de origen chino, ya sea legal o indocumentada. A través de un análisis basado en la aplicación de encuestas, en este artículo se estudian las principales causas y orígenes de los flujos migratorios de China a Puebla en tres distintos niveles de análisis: micro, meso y macro. El análisis tiene la finalidad de establecer posibles tendencias de la migración de China a Puebla en los próximos años. Un análisis detallado de los flujos migratorios de China a Puebla contribuirá a mejorar el entendimiento de este flujo migratorio en transición (sur-sur a norte-sur) y tener una visión más amplia de las razones de este fenómeno transnacional.

Palabras clave: China, México, Puebla, migración, migrantes.

Artículo recibido el 10 de noviembre de 2013 y dictaminado el 25 de febrero de 2014.

1. El doctor Gachúz Maya es maestro y doctor en Gobierno por la Universidad de Essex, Inglaterra; realizó una estancia posdoctoral de investigación en el Instituto de Asia de Harvard, Kennedy School of Government. Actualmente se desempeña como profesor de tiempo completo en la Academia de Relaciones Internacionales de la Benemérita Universidad Autónoma de Puebla. El autor agradece a Karla Esesarte, Yang Ziye y Gabriela Stefanoni por su apoyo logístico y administrativo en la realización de esta investigación. ORCID http://orcid.org/0000-0002-6770-0357 


\section{Abstract}

The objective of this paper is to analyze qualitatively the main reasons and causes of migration flows from China to the state of Puebla in the last 15 years. China has become one of the major trading partners of our country and the Chinese population in Mexico is one of the migrant communities with higher growth; recently, the state of Puebla has become one of the main destinations of Chinese migrations either legal or undocumented. Through an application based on survey analysis, this article examines the main causes and origins of migration flows from China to the state of Puebla in three different levels of analysis: micro, meso and macro. Analysis aims to establish possible migration trends from China to Puebla in the upcoming years. An analysis of the migration flows from China to Puebla will contribute to a better understanding of this transitory process (south-south to north-south) and take a broader view of the reasons for this transnational phenomenon.

Keywords: China, Mexico, Puebla, migration, migrants.

\section{Antecedentes}

El primer contacto comercial entre China y México data del siglo XVI, cuando los galeones de Manila navegaban a través del Océano Pacífico con mercancías y tripulación de China, acercando de esta forma a Oriente y Occidente. Estas embarcaciones continuaron realizando sus travesías por más de dos siglos y esto representó el primer acercamiento entre las dos culturas (Acosta y Zizumbo, 2011: 583-589).

El 14 de diciembre de 1899 China y México firmaron en Washington el "Tratado de amistad, comercio y navegación" que estipulaba que los nacionales de estos países eran libres de viajar, establecer residencia y hacer negocios; como parte del tratado también se establecieron oficinas diplomáticas y consulares de ambos países (Senado de la República, 1973).

Tras la firma del Tratado se alentó la migración China así como las relaciones comerciales entre ambos países. Según Eugenio Anguiano Roch, el Tratado de amistad puede ser considerado como la piedra angular de las corrientes de migración china a México. De acuerdo con Anguiano, desde 1899, con base en el tamaño de los flujos migratorios se pueden establecer siete fases principales de la migración china a México: primera fase de 1899 a1927, segunda de 1928 a 1945, tercera de 1946 a 1949, cuarta de 1959 a 
1971, quinta de 1972 a1981, sexta de 1982 al 2000 y séptima del 2000 a la actualidad (Anguiano, 2007: 25-50).

Con el gobierno de Porfirio Díaz, el Registro Nacional de Extranjeros se hizo obligatorio (Suprema Corte de Justicia, s/f: 251) y todos los inmigrantes que entraron a México a finales del siglo XIX comenzaron a registrarse de acuerdo con los parámetros de la también nueva Ley de Inmigración y la Ley de Naturalización.

De acuerdo con los datos del Archivo General de la Nación y del Registro Nacional de Extranjeros en México, el primer grupo de chinos coolies ${ }^{2}$ llegaron a México en 1875 y eran sólo 214; para el año 1910 el número se elevó a 3,442 (Ota, 1997: 75).

Los coolies provenían de las aldeas remotas de China y llegaban luego a Shanghai donde se embarcaban hacia diferentes países, incluido México. Su aspiración era siempre el trabajo arduo a fin de obtener suficiente dinero para invertir en un negocio que les permitiera vivir mejor, y algunos soñaban con volverse ricos (Ota, 1997).

En México, los trabajadores chinos llegaban al puerto de Veracruz, donde eran contratados por capataces para trabajar en los campos donde se realizan actividades agrícolas fundamentalmente. Uno de los destinos clásicos de estas tripulaciones fue Yucatán, donde había plantaciones de henequén. A pesar de ello, la mayoría de los chinos aprendieron a desarrollar otras ocupaciones (principalmente como pequeños comerciantes) y comenzaron a emigrar a otras partes del país (Ota, 1997). La migración china comenzaba a crecer y a expandirse en México. ${ }^{3}$

Xu Shicheng presenta cifras del censo de población de 1910 que muestra pruebas de la entrada de población china a México a través de las fronteras de Estados Unidos. Un número significativo $(10,127)$ llegó a Mexicali y otros se dirigieron a pequeños pueblos que hoy se conocen como la región de El Parador, cerca de San Felipe, Baja California; 13,263 llegaron a Sonora, 6,280

2. "El término coolie se utilizó predominantemente en los siglos XIX e inicios del XX para referirse a trabajadores manuales de origen asiático (algunos en situaciones de semiesclavitud), particularmente del sur de China" (Hu-Dehart, 1989: 91-116).

3. El caso de la "china poblana" tiene connotaciones particulares; de acuerdo con diversos analistas el origen de "Juana de Aragón" no tiene relación alguna con China; el personaje histórico de Puebla tiene en realidad como país de origen la India, posteriormente la mujer fue raptada por piratas portugueses y traída a la Nueva España para ser vendida como esclava. En Puebla se le llamó china poblana por su atuendo y sus características físicas diferentes a las locales. Véase Bailey, 1997: 38 y 39. 
se establecieron en Tamaulipas y 6,014 en la ciudad de México; otras ciudades que recibieron importantes flujos de migración china fueron Colima, Puebla, Nayarit, Chiapas y Jalisco (Yu, Shuzi, 1954: 55 y 56).

En los años que siguieron a la Revolución Mexicana hubo algunas protestas del Gobierno chino por los daños causados a sus nacionales durante el conflicto armado. En la década de 1920 el Gobierno mexicano y el Gobierno chino hicieron una revisión del Tratado de 1899. Se firmó un acuerdo que regulaba el modus vivendi de los inmigrantes chinos donde se establecía que la población china en México sería legalmente tratada con los mismos derechos que los ciudadanos mexicanos. Sin embargo, en esta etapa de turbulencia de México, el Tratado no se llevó a la práctica, por el contrario, muchos chinos fueron deportados, el gobierno de Álvaro Obregón fue acusado incluso de deportación violenta.

A finales de 1930, 70\% de la población china que vivía en México fue expulsada (Pardinas, 1982: 427). La deportación masiva se produjo sobre todo en Sonora y Sinaloa, pero grupos de chinos fueron deportados de todos los rincones del país. Algunos fueron deportados directamente a China, y muchos otros se trasladaron a Estados Unidos (Schiavone, 2009: 547-565).

En 1949, con el triunfo de la Revolución Socialista China y comienzos de la Guerra Fría, la relación de México y China mantuvo un perfil bajo debido principalmente a las políticas de migración de China; los flujos de migración china disminuyeron considerablemente (Connelly, 2010). En 1954 el Gobierno chino envió una carta al presidente Ruiz Cortines pidiendo el reconocimiento del Gobierno de la República Popular China y el apoyo para su entrada a las Naciones Unidas (ONU). La respuesta fue negativa y fue hasta finales de 1960 que México apoyó la entrada de China a las Naciones Unidas, por lo que en 1972 México estableció oficialmente relaciones diplomáticas con China. Desde entonces, todos los presidentes mexicanos han realizado visitas oficiales a China.

Entre 1980 y 1990 la migración china a México comenzó a crecer de nuevo, México aplicó reformas estructurales internas y estimuló el comercio exterior, lo cual favoreció la llegada de extranjeros al país. De forma paralela, la economía china comenzó a crecer a un ritmo acelerado, el país asiático empezó a implementar una serie de medidas para modernizar el aparato productivo y para incentivar la entrada de inversión extranjera. En 2009 China se convirtió en el segundo mayor socio comercial de México. El intercambio económico 
entre ambos países también trajo como consecuencia un aumento de los flujos migratorios chinos a México.

Actualmente, los ciudadanos chinos en México, junto con mexicanos con raíces chinas, representan una de las mayores comunidades de migrantes en algunas ciudades mexicanas. En cuanto a actividades económicas, los migrantes chinos en México son en su mayoría pequeños comerciantes, vendedores e importadores de productos chinos en México y muchos otros son dueños de restaurantes, algunos otros han incursionado en el mercado de los textiles. Adicionalmente, la paradiplomacia México-China se ha activado con la firma de acuerdos entre ciuda-
Actualmente la población china en México representa una de las comunidades más grandes de migrantes en México, China se ha convertido en uno de los principales socios comerciales de nuestro país y la relación política entre ambos países también ha mejorado en los últimos 10 años des y provincias chinas con ciudades y estados mexicanos como: QingdaoAcapulco (1985), Nanchang-Toluca, Nanjing-Mexicali (1988), ShenyangMonterrey (1991), Panjin-Tijuana (1995), Shijiazhuang-Querétaro (1997), Shanghai-Jalisco (1998), Hengshui-Tuiltitlán (2002), Xiamen-Guadalajara (2003), provincia de Jiangsu-Baja California (2006), provincia de HenanHidalgo (2006) (Embajada de la República Popular China en México, 2011).

La creciente importancia de la relación México-China hace que este tema sea cada vez más relevante. Actualmente la población china en México representa una de las comunidades más grandes de migrantes en México, China se ha convertido en uno de los principales socios comerciales de nuestro país y la relación política entre ambos países también ha mejorado en los últimos 10 años. Un análisis detallado de los flujos migratorios de China a México contribuirá a mejorar la relación entre ambos países y permitirá propiciar una dinámica de entendimiento y cooperación en distintos ámbitos.

\section{Marco teórico}

Existen diferentes enfoques teóricos para analizar las migraciones internacionales. Teorías basadas en la economía neoclásica ven la migración como un 
componente de mercados racionales que tiende al equilibrio y es impulsado por la toma de decisiones económica (racional) de los migrantes individuales o de sus familias. Estas teorías asociadas tienen sus antecedentes en el modelo de comportamiento racional de Gary Becker, rational choice theory $y^{4}$ evolucionan en lo que ahora se denominan teorías push-pull de las migraciones internacionales, mismas que explican los flujos migratorios en términos de oferta y demanda de mano de obra entre emisores y receptores; la nueva literatura de migración de mano de obra se centra en la toma de decisiones ${ }^{5}$ de migración en términos de maximización o protección del ingreso a nivel micro (Stark y Bloom, 1985: 173-178).

En esta perspectiva se acentúan los así llamados "factores de expulsión" (push factors en la terminología académica) que "empujan" a los migrantes a dejar sus respectivas regiones o países debido a factores internos identificables como persecución, falta de libertades civiles, guerras, dificultades económicas, persecuciones religiosas, desastres medioambientales, etc. De la misma manera están los enfoques que acentúan los "factores de atracción" (pull factors) que atraen a los migrantes hacia determinadas regiones o países con base en variables como mejores condiciones de vida, más libertades civiles, menos competencia económica y social, salarios más altos, libertad de religión, democracia, paz, mejores condiciones de vida y perspectiva de un futuro mejor.

Por el contrario, los enfoques "histórico-estructurales" o neomarxistas analizan a los migrantes no como actores económicos libres y racionales que operan dentro de un mercado de oferta y demanda, sino como trabajadores cuyo movimiento está determinado por las estructuras de explotación y procesos de acumulación capitalista. ${ }^{6}$ Rick Wolff, por ejemplo, señala en este sentido que los procesos migratorios ocurren como características del sistema cuando estos procesos ayudan a resolver contradicciones sociales internas y especialmente de clase en cada uno de los países (emisores y receptores), una serie de contradicciones obliga a un grupo de personas a salir de un país, así como otro país con otro grupo de contradicciones atrae tales flujos migratorios. Finalmente, mientras la migración "resuelve" algunas contradicciones sociales, de manera paralela agrava o genera otras (Wolff, 2006).

\footnotetext{
4. Véase Becker, 1976.

5. Véase De Jong, Gordon F., y Gardner, Robert W., 1981. Véase también Portes, 1995.

6. Véase Baran, 1989.
} 
Críticas de las teorías basadas en aspectos económicos se han centrado en la visión reduccionista que estas teorías proponen: migrantes y sus familias como sujetos atomizados sujetos a decisiones económicas racionales. De cualquier manera, en la búsqueda de teorías generales que expliquen la migración y sus consecuencias, ha habido una tendencia a abstraer la "migración económica” de la migración política, social, cultural, institucional e histórica. Como consecuencia de ello, estos enfoques han fallado en dar explicaciones generalmente aplicables y predictivas que proporcionen una explicación adecuada del flujo de migrantes. ${ }^{7}$ Por ejemplo la teoría de push and pull ha sido criticada porque falla al explicar por qué sólo algunos sujetos tienden a migrar, no explica con precisión quiénes son esas personas, la razón por la que eligen migrar, mientras que otros prefieren no hacerlo; las teorías neoclásicas parecen estar limitadas por la suposición de que los migrantes tienen conocimiento acerca de los costos y beneficios de la migración.

En el caso de la migración china a otros países, ${ }^{8}$ la literatura existente muestra que las corrientes de migración se ven determinadas por las relaciones económicas, políticas, familiares y comunales que influyen y median la interacción dinámica entre la estructura y la acción. Elementos críticos de la teoría racional así como de los enfoques estructurales son necesarios para identificar los principales patrones de comportamiento de los flujos migratorios chinos a México; es importante señalar que la migración china a México no puede considerarse enteramente migración sur-sur, la china es ahora la segunda economía más grande del mundo (CIA) y varios pronósticos indican que será la mayor economía dentro de 15 años.

La migración México-China se encuentra en transición hacia un modelo de migración de norte a sur; por lo tanto, ni los factores de push and pull theories ni los enfoques estructurales (centro-periferia) pueden quedar fuera del análisis; a partir de elementos clave de ambas perspectivas teóricas podremos obtener una visión más amplia del problema, y nos permitirá conocer con más detalle las causas de la migración china en Puebla.

Las hipótesis de esta investigación se basan en la suposición de que la migración china a México está principalmente relacionada con motivos económicos; sin embargo, otros factores como los lazos familiares e incluso la corrupción existente en ambos países alientan estos flujos migratorios. Esta

7. Véase Gold, 2005.

8. Véanse Tan Chee, 2007; Kwok, 2005; Nyiri, y Saveliev, 2003; B. Chang, 2011; Guofuo, 2006. 
investigación contribuirá a analizar con detalle cada uno de estos factores y coadyuvará a establecer una perspectiva más clara sobre este tema.

\section{Metodología}

Tres niveles de análisis: muestreo micro/macro/meso y clúster

Los patrones y la dinámica de la migración son complejos, variados y no estáticos. Por medio de los diferentes niveles de análisis evaluaremos los flujos de migración internacional de manera más precisa. Para los propósitos de nuestra investigación utilizaremos tres niveles de análisis: a) el grado de libertad o autonomía de un migrante potencial, o el nivel micro; éste es el grado de libertad que los migrantes tienen para decidir migrar o quedarse en su país de origen; b) las estructuras político-económico-culturales en el país de origen, el país de destino y el sistema mundial, es decir el nivel macro; en este sentido, el análisis se enfoca en las estructuras transnacionales y las relaciones comerciales entre Estados-nación, y c) el conjunto de vínculos sociales y simbólicos entre migrantes y los recursos inherentes a estas relaciones constituyen el nivel meso. Estos factores se refieren a la estructura, fuerza y densidad de lazos sociales, por un lado, y su contenido, por otro. Esta dimensión relacional analiza los lazos sociales y simbólicos entre stayers y migrantes con unidades y redes en las áreas de origen y de destino, y las relaciones entre los actores colectivos pertinentes, entre otros aspectos, grupos familiares, hogares, grupos religiosos, comunidades étnicas, etc. También contiene los recursos inherentes a esos vínculos que faciliten la cooperación, es decir, capital social.

A través de indicadores específicos en las preguntas se analizaron los tres niveles de análisis usando el sistema de encuestas (cara a cara) a inmigrantes chinos en México. El diseño de cuestionarios se enfocó en las variables macro, meso y micro como base para analizar los principales factores que explican la migración china en México. El cuestionario se redactó en chino mandarín y español para facilitar su comprensión, ya que gran parte de los migrantes en Puebla aún no dominan el español, ${ }^{9}$ y también se revisó en una etapa de focus groups con tres ciudadanos chinos que aportaron opiniones e hicieron algunos

9. Las encuestas se aplicaron además con ayuda de Yang Ziye (una traductora nativa de China) y de Karla Esesarte, esto con la finalidad de lograr mayor confianza, empatía y objetividad en las respuestas de los cuestionarios. 
Cuadro 1

Tres niveles de análisis: micro, meso y macro

\begin{tabular}{|c|c|c|}
\hline $\begin{array}{l}\text { Micro } \\
\text { Valores o deseos y } \\
\text { expectativas }\end{array}$ & $\begin{array}{l}\text { Meso } \\
\text { Colectivos y redes sociales }\end{array}$ & $\begin{array}{l}\text { Macro } \\
\text { Estructuras de oportunidad a } \\
\text { nivel macro }\end{array}$ \\
\hline $\begin{array}{l}\text { Valores individuales y } \\
\text { esperanza de mejor vida } \\
\text { - Mejorar y asegurar la } \\
\text { supervivencia, riqueza, } \\
\text { Estado, comodidad, es- } \\
\text { timulación. Autonomía, } \\
\text { la afiliación y la moralidad }\end{array}$ & $\begin{array}{l}\text { Lazos sociales } \\
\text { - Hogares y familias fuertes } \\
\text { lazos } \\
\text { - Migrantes y stayers: } \\
\text { Vinculos simbólicos, } \\
\text { organizaciones familiares, } \\
\text { étnicas, nacionales, políticas } \\
\text { y religiosas, comunidades } \\
\text { simbólicas } \\
\text { Contenido de lazos: } \\
\text { transacciones } \\
\text { Las obligaciones, } \\
\text { reciprocidad y solidaridad: } \\
\text { información, control y } \\
\text { acceso a recursos de otros }\end{array}$ & $\begin{array}{l}\text { Economía } \\
\text { Ingreso y desempleo } \\
\text { Política } \\
\text { - Reglamentos de mo- } \\
\text { vilidad espacial a través } \\
\text { de los Estados-nación y } \\
\text { regímenes internaciona- } \\
\text { les; } \\
\text { - Configuración cultural los } \\
\text { conflictos religiosos, ét- } \\
\text { nicos, nacionales y repre- } \\
\text { sión política } \\
\text { - Discursos y normas domi- } \\
\text { nantes } \\
\text { Demografía y ecología } \\
\text { - Crecimiento de la po- } \\
\text { blación: } \\
\text { - Disponibilidad de recur- } \\
\text { sos } \\
\text { - Nivel de tecnología }\end{array}$ \\
\hline
\end{tabular}

Fuente: Collinson, Sarah, The Political Economy of Migration Processes, and Agenda for Migration Research and Analysis, International Migrations Institute, Working papers, 2009, paper 12, University of Oxford.

ajustes en las preguntas para lograr mejor índice de respuesta y entendimiento de los reactivos. El cuestionario que se aplicó tiene las siguientes preguntas:

1. ¿Cuál es su ciudad de origen? 请问您来自中国什么地方?

2. ¿En qué otras ciudades de China vivió antes de venir a México? 请问在 老墨西哥之前您在中国其他城市居住过吗?

3. ¿Hace cuánto que vive en México? ¿Qué ruta siguió desde China para venir a México? (barco, avión, ¿fue antes a otro país?, etc.). 请问您是什 么时候老墨西哥的, 是乘坐什么交通工具呢? 
4. ¿Cuáles son las razones por las que vino a vivir a México? 请问您为什么 选择移民, 为什么选择墨西哥呢?

5. ¿Con quién vive en México (familiares, amigos, conocidos, etc.)? 在墨西 哥您跟谁一起居住呢?

6. ¿A cuántos chinos conoce en Puebla? ¿Cuál cree que sea el número de chinos viviendo en Puebla?

您认识多少在普埃布拉的中国人呢? 您认为在普埃布拉大概有多少 中国人呢?

7. ¿Le gusta vivir en México? ¿Cuál es la diferencia de vivir en México y en China? 您喜欢这里的生活吗? 和在中国有什么不同吗?

8. China ha tenido un gran crecimiento en la última década, ¿por qué decidió venir a vivir a México? 问您为什么选择移民, 为什么选择墨西哥呢?

9. ¿Qué no le gusta de vivir en México? 墨西哥有什么地方是您不喜欢的 吗?

10. ¿Cuáles son sus planes futuros? (quedarse a vivir permanentemente en México, traer a más familiares, irse a otro país, estado, regresar a China, etc.) 您未来有什么计划吗?

11. ¿Conoce alguna asociación de ciudadanos chinos en Puebla? 请问你知道 在Puebla 有没有一些中国人的组织?

12. ¿Tiene algún apoyo del Gobierno de China para vivir en México? 您为什 么选择在这开饭店 中国政府对于？您这种选择有什么支持吗?

El Instituto Nacional de Migración, por conducto del Centro de Estudios Migratorios ${ }^{10}$ ofrece datos sobre las migraciones legales e ilegales de ciudadanos chinos a México; lamentablemente no existe una lista completa y precisa de la población. El INM calcula que hay 10,247 chinos residentes en México; sin embargo, no existen datos estadísticos sobre la población en cada uno de los estados de México, no existe tampoco una estimación oficial de este número (Instituto Nacional de Migración, 2012).

El muestreo multifase (multistage sampling) es útil en estos casos. Un diseño de muestreo multifase implica la selección de muestras de un universo registrado como una selección principal, y posteriormente a partir de este universo se hace una selección aleatoria.

10. Para mayor información consultar http://www.inm.gob.mx/index.php/page/Estimacion_de_ Poblacion 
De un total de 42 negocios chinos en la capital poblana (universo registrado), se aplicaron 30 encuestas (cara a cara). Cada encuesta se aplicó en un lapso de una hora (dependiendo de las respuestas de los entrevistados y se buscaron obtener detalles adicionales con base en respuestas o temas específicos que los entrevistados dieron a conocer.

Con base en la información obtenida, se encontró que la mayoría de los inmigrantes chinos en Puebla sólo habían vivido en China antes; la mayoría vino por avión y por barco a México directamente; no hay asociaciones de chinos en Puebla y la comunidad en general está dispersa. Las siete preguntas restantes, por su complejidad, las analizaremos con mayor detalle a continuación.

\section{Gráfica 1}

Ciudad de origen

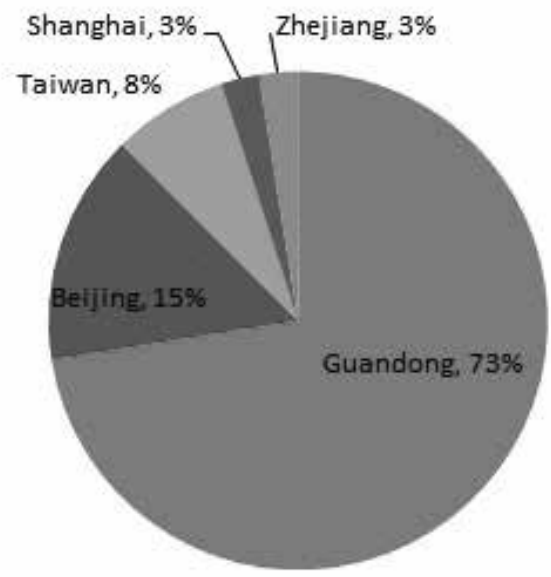

Como podemos observar, en las respuestas obtenidas respecto a ciudad de origen, encontramos que la mayor parte de la población que habita en Puebla proviene de la provincia de Guangdong; dentro de esta provincia hay otras ciudades importantes, entre las que destacan Shantou, Shenzhen, Donguan y Maoming; esta ciudad se localiza al sur del país, colinda con el Río de las Perlas (Pearl River), uno de los ríos más grandes de China, tiene una extensión de 186,000 kilómetros cuadrados y una población de más de 95 millones de habitantes (2012), y aunque es una de las regiones con más crecimiento y más prósperas del país, también es una de las ciudades con más sobrepoblación 
y expulsión de migrantes hacia el exterior. Su proximidad con Hong Kong y Macao le otorga ciertas ventajas en cuanto a intercambio comercial. Sin embargo, debido al marco regulatorio actual, la población de la provincia de Guangdong no puede emigrar a estas ciudades sin un permiso especial (CUAL). La encuesta también refleja que un número importante de habitantes de la capital, Beijing y de Shanghai están haciendo negocios en México y su estancia en el país tiene características predominantemente temporales.

Respecto a la pregunta 2. China ha tenido un gran crecimiento en la última década, ¿por qué decidió venir a vivir a México? 请问您为什么选择移民, 为什么选择墨西哥呢? Los resultados fueron los siguientes:

\section{Gráfica 2}

¿Por qué decidió establecer su residencia en México?

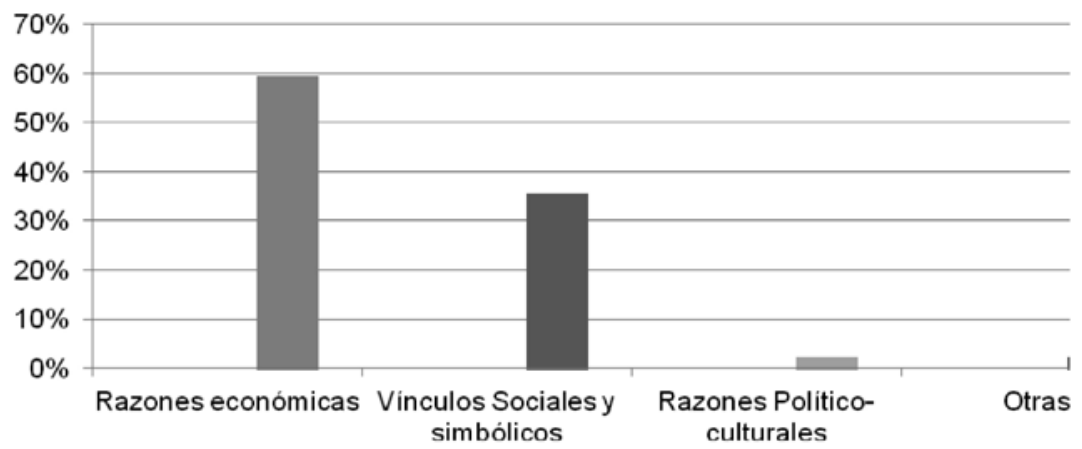

Encontramos en las respuestas que casi $60 \%$ de la población china en Puebla está en México debido a razones económicas (búsqueda de empleo, trabajo, desarrollo de negocios, etc.); posteriormente un número importante de encuestados (casi 30\%) afirman que están en Puebla debido a vínculos familiares y de amigos, y un número muy pequeño afirman que vienen a México buscando mejores condiciones en cuanto a derechos humanos, libertades civiles, etcétera. Los factores de atracción (pull factors) explican el comportamiento de los migrantes chinos a México con base en variables como mejores condiciones de vida, más libertades civiles, menos competencia económica y social, salarios más altos, libertad de religión, democracia, paz, mejores condiciones de vida y perspectiva de un futuro mejor. Sin embargo, como podemos observar en la siguiente gráfica, otro número importante de 
migrantes se establece en México por razones de vínculos familiares o de amistades.

No podemos ignorar, sin embargo, las condiciones internas de China y las causas que determinan la decisión de ciudadanos chinos de emigrar a otros países, en este caso México. El enfoque "histórico-estructural" es útil en este aspecto ya que analiza a los migrantes no como actores económicos libres y racionales que operan dentro de un mercado de oferta y demanda, sino como trabajadores cuyo movimiento está determinado por las estructuras de explotación y procesos de acumulación capitalista (Baran,1989).

Como mencionamos anteriormente, con base en esta perspectiva los procesos migratorios ocurren como características del sistema cuando estos procesos ayudan a resolver contradicciones sociales internas y especialmente de clase en cada uno de los países (emisores y receptores), una serie de contradicciones obliga a un grupo de personas a salir de un país así como otro país con otro grupo de contradicciones atrae tales flujos migratorios. Finalmente, mientras la migración "resuelve" algunas contradicciones sociales de manera paralela, agrava o genera otras (Wolff, 2006).

La sobrepoblación, saturación del mercado de trabajo, falta de oportunidades de trabajo y falta de libertades civiles son también razones de peso para explicar los flujos migratorios de China a México.

La pregunta siguiente: ¿con quién vive en México (familiares, amigos, conocidos, etc.) 在墨西哥您跟谁一起居住呢?

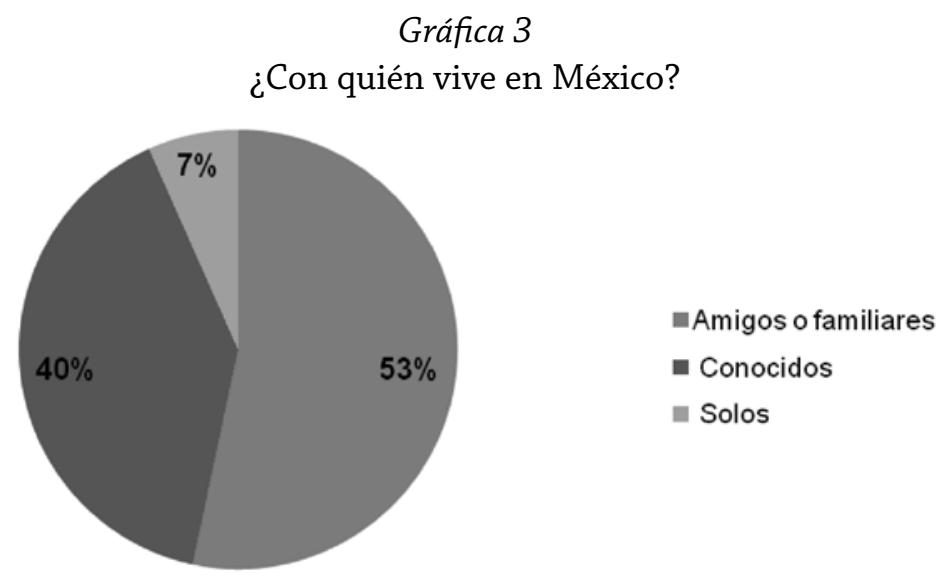


Las respuestas indican que la mayor parte los encuestados viven en México con amigos o familiares (más de 50\%); después encontramos que $40 \%$ viven con conocidos y un número pequeño $(7 \%)$ viven solos, tienen casa propia o renta y no comparten vivienda con nadie.

A pesar de que existe un vínculo familiar fuerte entre los migrantes chinos y los residentes chinos en México, en general se observa que la población china en Puebla desconoce el número de compatriotas suyos en el estado. En la pregunta siguiente: ¿a cuántos chinos conoce en Puebla? 您认识多少 在普埃布拉的中国人呢? 您认为在普埃布拉大概有多少中国人呢? Las respuestas fueron las siguientes:

\section{Gráfica 4}

¿A cuántas personas de origen chino conoce en Puebla?

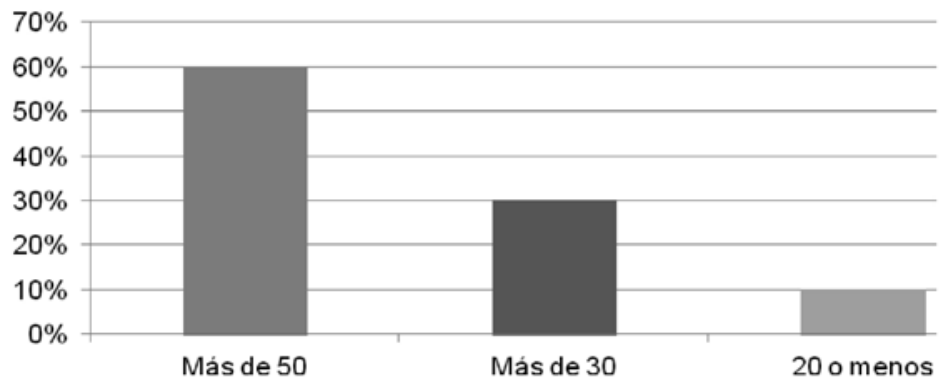

El vínculo social en la población china en Puebla (más allá del vínculo familiar) parece estar disperso, y los habitantes chinos en Puebla manifiestan no conocer un número importante de personas de su mismo origen en el estado. El 60\% contestan que conocen a más de 50, 30\% más de 30 y 10\% menos de 20 personas.

La siguiente pregunta tiene que ver con las condiciones que la población china encuentra en Puebla, particularmente si consideran que Puebla es un buen lugar para establecer residencia permanente y si encuentran atractivo, en términos generales, el país. Las respuestas fueron las siguientes: 


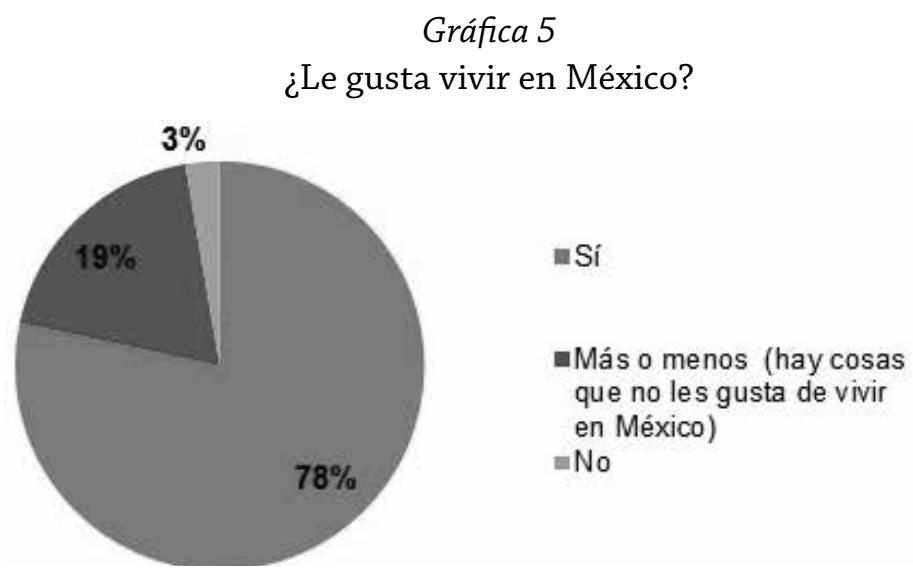

La mayoría de los encuestados afirman que les gusta vivir en México (78\%), aunque hay un número importante (19\%) que no están convencidos al respecto y hay cosas que no les gusta de vivir en el país; $3 \%$ afirman que no les gusta vivir en Puebla. El siguiente indicador tiene como objetivo conocer cuáles son los aspectos que a la población china en Puebla no les gusta respecto a vivir en México; la pregunta que se plantea es la siguiente: ¿qué aspectos no le gustan de vivir en México? 墨西哥有什么地方是您不喜欢的吗?

\section{Gráfica 6}

¿Qué aspectos no le gustan de vivir en México?

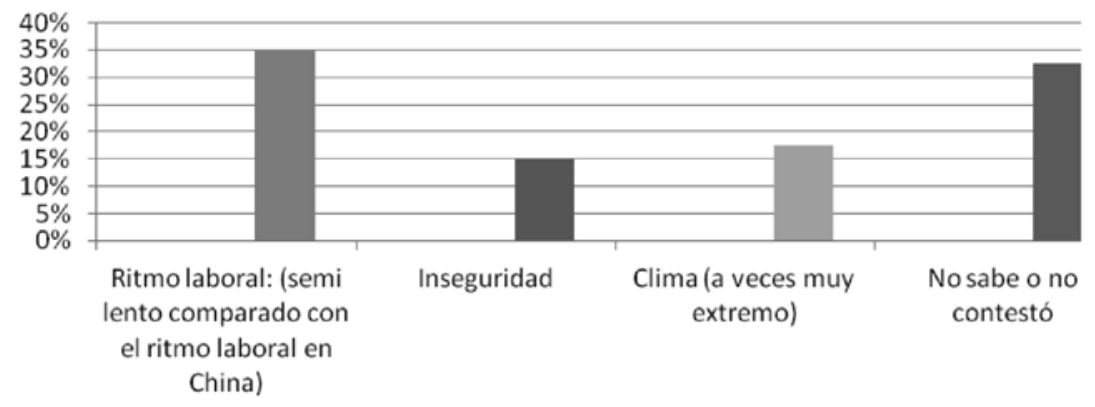

Como podemos observar, el ritmo laboral (semilento comparado con el de China) es el aspecto que más desagrada a la población china viviendo en Puebla; de acuerdo con las respuestas, esto se debe sobre todo al desempeño 
de trabajo de empleados locales y en general el ritmo de trabajo en el estado comparado con los estándares de trabajo más intensivo en China; después viene el tema de la seguridad que impacta en la percepción de los encuestados y aunque algunos enfatizaron que no es precisamente la inseguridad en Puebla a la que se refieren, sí hay una percepción generalizada de que el país presenta problemas serios de seguridad comparados con China.

Factores culturales intervienen en esta percepción generalizada de los residentes chinos en Puebla; el sistema económico chino también influye en esta percepción, ya que en general el Estado chino tiene un control más estricto sobre empresas públicas y privadas y el país sigue orientando su política económica con base en metas o "planes quinquenales".

El conocer los objetivos de la población china en Puebla es una de las prioridades de este estudio; el saber con detalle cuál es el plan de vida de los entrevistados a mediano y largo plazos es el objetivo principal de la siguiente pregunta: ¿cuáles son sus planes futuros? 您未来有什么计划吗? Las respuestas fueron las siguientes:

\section{Gráfica 7}

¿Cuáles son sus planes futuros?

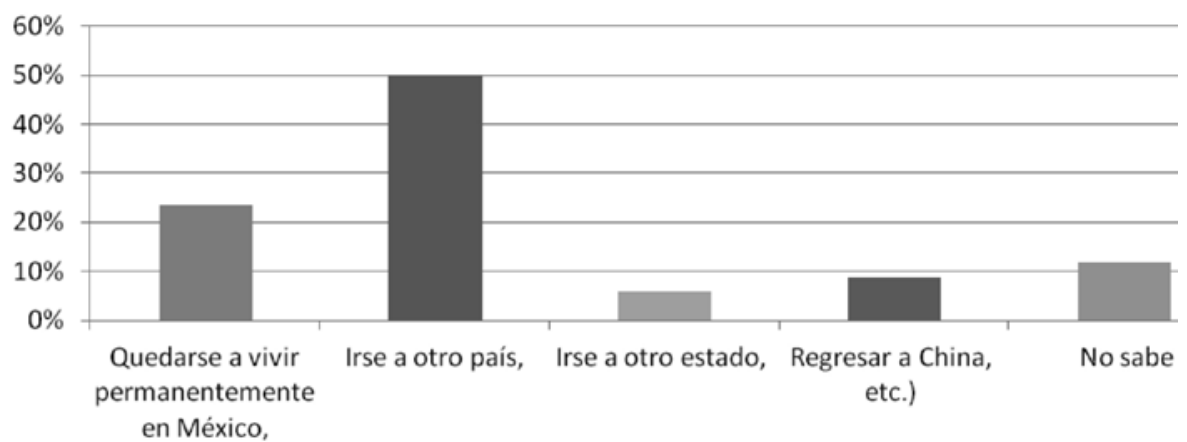

Las respuestas indican que sólo 20\% de la población china en Puebla tiene planes de quedarse en México, la mayoría (casi 50\%) se encuentran trabajando de manera temporal en México (aunque esta estancia se extienda por más de 10 años), ahorrar una cantidad significativa de dinero que les permita después establecerse en otros países (principalmente Estados Unidos o Canadá). 
Un 5\% tiene planeado cambiar de residencia a una ciudad más grande como el Distrito Federal, casi $10 \%$ tiene pensado en el mediano o largo plazo regresar a China y otro $10 \%$ no sabe aún qué hará en el mediano y largo plazos.

Los resultados nos indican que gran parte de la población china en Puebla es en realidad población flotante, aun cuando la mayoría tiene tres años o más años de vivir en México. El objetivo de más de $50 \%$ de los entrevistados es reunirse con amigos o familiares o iniciar una nueva vida principalmente en ciudades de Estados Unidos o Canadá, aun sin obtener la residencia legal en esos países, es decir, serían potencialmente población indocumentada en esos países.

\section{Conclusiones}

El análisis estadístico realizado muestra que los patrones y la dinámica de la migración son complejos, variados y no estáticos; es evidente también que la comunidad china en Puebla presenta características diferentes al de otros grupos de extranjeros en el estado (Alemania, España, Líbano, etcétera).

Una combinación de la teoría racional económica con enfoques estructurales es útil para identificar los principales patrones de comportamiento de los flujos migratorios chinos a México; la migración México-China se encuentra en transición hacia un modelo de migración sur-sur, a una migración de norte a sur; por lo tanto, los factores de push and pull theories así como un enfoque estructural (centro-periferia) se complementan dentro del análisis. Un análisis de ambas teorías permite a su vez tener una visión más amplia de la situación de los flujos migratorios, lo cual hace más integral el estudio para conocer con más detalle las causas de la migración china en Puebla.

Como pudimos observar, los habitantes chinos en el estado de Puebla son una población altamente polarizada en cuanto a origen (predominantemente de la provincia de Guangdong), cuya principal motivación al establecer su residencia en México es económica (hacer negocios, conseguir empleo, etc.); en la última década la gran mayoría viene al país porque familiares y amigos ya tienen tiempo de residir en México; la mayor parte del grupo de chinos viviendo en México tiene como meta a mediano y largo plazos ahorrar lo suficiente y tratar de emigrar a otros países (principalmente Estados Unidos y Canadá), aunque algunos deciden quedarse y sus hijos adquieren la nacionalidad mexicana. Los migrantes chinos en Puebla manifiestan su intención de migrar a otros países (de alto ingreso per cápita y desarrollo económico) 
pero se observa de manera generalizada que la estancia "temporal" se extiende indefinidamente y algunos finalmente deciden radicar de forma permanente en México.

La tendencia en el crecimiento de flujo de migrantes chinos al estado de Puebla dependerá en gran medida de las condiciones económicas y de seguridad que ofrece el país y también del creciente lazo familiar y de amistad que se presenta entre los migrantes chinos que deciden quedarse a vivir permanentemente en el país: los factores internos y aquellos que buscan salir de China para buscar oportunidades económicas en otros países. No podemos ignorar, sin embargo, las condiciones internas en China que motivan la migración de ciudadanos chinos a otros países; como lo establece en enfoque histórico-estructural, los procesos migratorios ocurren como características del sistema cuando estos procesos ayudan a resolver contradicciones sociales internas y especialmente de clase en cada uno de los países (emisores y receptores), una serie de contradicciones obliga a un grupo de personas a salir de un país así como otro país con otro grupo de contradicciones atrae tales flujos migratorios.

Finalmente, mientras que la migración "resuelve" algunas contradicciones sociales, de manera paralela agrava o genera otras (Wolff, 2006). Como ya mencionamos, en el caso de China algunos factores como sobrepoblación, saturación del mercado de trabajo, falta de oportunidades de trabajo y falta de libertades civiles son también razones de peso para explicar los flujos migratorios de China a México.

\section{Referencias bibliográficas}

Acosta Cázares, Jorge Antonio, y Zizumbo Ramírez, Haydee (2011), "Breve análisis de la migración china a México", Revista Exploratoris Observatorio de la Realidad Global, núm. 22, pp. 583-589, disponible en http://academiajournals.com/downloads/AcostaX11.pdf

Anguiano Roch, Eugenio (2007), "Relaciones México-China en su perspectiva histórica”, en Dussel Peters, Enrique, y Trápaga Delfín, Yolanda. (eds.), China y México: Implicaciones de una nueva relación, México: La Jornada; ITESM; Cechimex y UNAM, pp. 25-50.

Autor no especificado (2008), "La inmigración china", Enciclopedia de Sonora, disponible en: http://archive.is/f1tqb 
B. Chang, Felix (ed.) (2011), Chinese Migrants in Russia, Central Asia and Eastern Europe, Reino Unido: Routledge (Routledge Contemporary Russia and Eastern Europe Series).

Bailey, Gauvin A. (1997), "A Mughal Princess in Baroque New Spain. Catarina de San Juan (1606-1688), the China Poblana", Anales del Instituto de Investigaciones Estéticas de la Universidad Nacional Autónoma de México, núm. 71, pp. 38-39.

Baran, Paul (1989), Political economy of growth, Estados Unidos: Monthly Review Press.

Becker, Gary S. (1976), The Economic Approach to Human Behavior, Estados Unidos: University of Chicago Press.

Central Intelligence Agency (CIA) "China", World Fact Book, disponible en https://www.cia.gov/library/publications/the-world-factbook/geos/ ch.html

Collinson, Sarah (2009), "The political economy of migration processes, and agenda for migration, research and analysis", International Migrations Institute Working Papers, núm. 12, University of Oxford.

Connelly, Marisela (2010), "Las relaciones de China y México en su contexto histórico”, Escenarios XXI, año I, 5(6), disponible en http://www.escenarios21.com/2010/0039.html

Corte Suprema de Justicia (s/f), Jurisprudencia constitucional: Recopilación de las sentencias de constitucionalidad dictadas entre 1890 y 1990 por la Corte de Casación y la Corte Plena [on line], Costa Rica: Universidad Estatal a Distancia.

De Jong, Gordon F., y Gardner, Robert W. (1981), Migration Decision Making. Multidisciplinary Approaches to Microlevel Studies in Developed and Developing Countries. Nueva York: Pergamon.

Dehesa, Guillermo (2008), Comprender la inmigración, Madrid: Alianza Editorial.

Department of Economic and Social Affairs (2011), Trends in international migrant stock: Migrants by age and sex, ONU, disponible en http://www. un.org/esa/population/migration/cd-rom\%20documentation_un_migrantstock_2010.pdf

Durand, Jorge (agosto 28, 2011), "La inmigración china", La Jornada, disponible en http://www.jornada.unam.mx/2011/08/28/opinion/018a1pol

Embajada de la República Popular China en México (2011), China-México, disponible en http://www.embajadachina.org.mx/esp/zmgx/t44249.htm 
Gold, S. J. (2005), "Migrant Networks: A Summary and Critique of Relational Approaches on International Migration", en Romero y Margolis (eds.), The Blackwell Companion of Social Inequalities, Malden Blackwell.

Guofuo, Liu (2006), The Right to Leave and Return and Chinese Migration Law, Estados Unidos: Martinus Nijhoff Publishers.

Hu-Dehart, Evelyn (1989), "Coolies, Shopkeepers, Pioneers: The Chinese of Mexico and Peru (1849-1930)", Amerasia Journal, 15(2), pp. 91-116.

Instituto Nacional de Migración (2012), Síntesis 2012, estadística migratoria, disponible en http://www.inm.gob.mx/estadisticas/Sintesis_Grafica/2012/Sintesis2012.pdf

Klugman, Jeni (coord.) (2009), "Superando barreras: Movilidad y desarrollo humanos", Programa de las Naciones Unidas para el Desarrollo, disponible en http://hdr.undp.org/sites/default/files/hdr_2009_es_summary.pdf

Kwok, BunChan (2005), Migration, Ethnic Relations and Chinese Business, Reino Unido: Routledge.

McDowell, C., y de Haan, A. (1997), "Migration and Sustainable Livelihoods, a Critical Review of Literature”, IDS working paper, núm. 65, Reino Unido: Institute of Development Studies.

Muthén, B. O. (2002), "Beyond SEM: General Latent-Variable Modeling", Behaviormetrika, núm. 29, pp. 81-117.

Nyiri, Pal, y Saveliev, Igor (2003), Globalizing Chinese Migration: Trends in Europe and Asia, Research in Migration and Ethnic Relations), Reino Unido: Ashgate Pub Ltd.

OCDE (2 de agosto, 2011), Inmigrantes en México, censo OCDE 2000.

Pardinas, Felipe (1982), Relaciones diplomáticas entre México y China, 18981948, vol. I del Archivo Histórico Diplomático Mexicano, México: Secretaría de Relaciones Exteriores.

Portes, A. (ed.) (1995), The Economic Sociology of Immigration: Essays on Networks, Ethnicity, and Entrepreneurship. Nueva York: Russell Sage Foundation.

Resnick, S. (1990), "China Today and Class Analysis", Rethinking Marxism, pp. 157-163.

Schiavone Camacho, Julia María (2009), “Crossing Boundaries, Claiming a Homeland: The Mexican Chinese Transpacific Journey to Becoming Mexican, 1930s-1960s”, Pacific Historical Review, 78(4), Berkeley, pp. 547-565. Secretaría de Relaciones Exteriores (1973), "Discurso del C. Presidente de la República Licenciado Luis Echeverría Álvarez ante la Asamblea General 
de la ONU, en el XXVI periodo de sesiones celebrado el 5 de octubre de 1971", Memoria de la Secretaría de Relaciones Exteriores, 1 de septiembre de 1971 al 31 de agosto de 1972, México, p. 222.

Senado de la República (México) (1973), Tratados ratificados y convenios ejecutivos celebrados por México, México: Senado de la República.

Skeldon, R. (1992), "International Migration within and from the East and Southeast Asian Region: A Review Essay”, Asian Pacific Migration, 1(1), pp. 19-63.

Stark, y Bloom (1985), "The new economics of labor migration", The American Economic Review, pp. 173-178.

Tan Chee, Beng (2007), Chinese Overseas: Migration, Research and Documentation, China: The Chinese University Press.

Trápaga Delfín, Yolanda (2007), "China y México: Implicaciones de una nueva relación”, La Jornada, pp. 55 y 56.

Van Tubergen, F., Maas, I., y Flap, H. (2004), "The Economic Incorporation of Immigrants in 18 Western Societies: Origin, Destination, and Community Effects", American Sociological Review, núm. 67, pp. 704-727.

Waldinger, R. (2007), "Did Manufacturing Matter? The Experience of Yesterday's Second Generation: A Reassessment", International Migration Review, 41(1), pp. 3-39.

Wolff, Rick (mayo 10, 2006), "Immigration and Class", Rethinking Marxism, disponible en: http://rmsplash.blogspot.mx/2006/05/immigration-andclass.html

Wooldridge, J. M. (2002), Econometric Analysis of Cross Section and Panel Data, Cambridge/Londres: MIT Press.

Yu, Shuzi (1954), Historia de los chinos en México, Taipei: Hawái Wenku. 


\section{YUSUKE MURAKAMI}
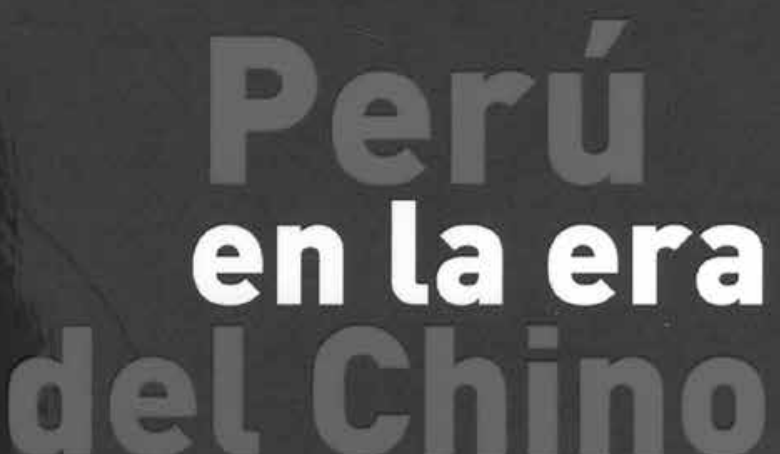

La política no institucionalizada y el pueblo en busca de un salvador

\section{Segunda edición}

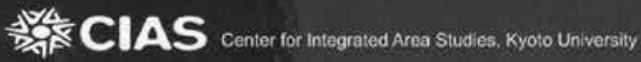

IEP Instituto de Estudios Peruanos 